\title{
Nonlinear Stability and Convergence of Two-Step Runge-Kutta Methods for Neutral Delay Differential Equations
}

\author{
Haiyan Yuan, ${ }^{1}$ Cheng Song, ${ }^{2}$ and Peichen Wang ${ }^{1}$ \\ ${ }^{1}$ Department of Mathematics, Heilongjiang Institute of Technology, Harbin 150050, China \\ ${ }^{2}$ School of Management, Harbin University of Commerce, Harbin 150028, China \\ Correspondence should be addressed to Haiyan Yuan; yhy82_47@163.com
}

Received 29 January 2013; Revised 4 April 2013; Accepted 4 April 2013

Academic Editor: Hamid Reza Karimi

Copyright (C) 2013 Haiyan Yuan et al. This is an open access article distributed under the Creative Commons Attribution License, which permits unrestricted use, distribution, and reproduction in any medium, provided the original work is properly cited.

\begin{abstract}
This paper is devoted to the stability and convergence analysis of the two-step Runge-Kutta (TSRK) methods with the Lagrange interpolation of the numerical solution for nonlinear neutral delay differential equations. Nonlinear stability and $\mathrm{D}$-convergence are introduced and proved. We discuss the $\operatorname{GR}(l)$-stability, $\operatorname{GAR}(l)$-stability, and the weak $\operatorname{GAR}(l)$-stability on the basis of $(k, l)$ algebraically stable of the TSRK methods; we also discuss the D-convergence properties of TSRK methods with a restricted type of interpolation procedure.
\end{abstract}

\section{Introduction}

Neutral delay differential equations (NDDEs) arise in a variety of fields as biology, economy, control theory, and electrodynamics (see, e.g., [1-5]). The stability and convergence properties of numerical methods for linear NDDEs have been widely researched by many authors (see, e.g., [611]). For the case of nonlinear delay differential equations, this kind of methodology had been first introduced by Bellen and Zennaro [12] and Torelli [13] and then developed by Torelli [14], Bellen [15], and Zennaro [16, 17]. In 1997, Koto proved the asymptotic stability of natural Runge-Kutta method for a class of nonlinear delay differential equations in [18]. Bellen et al. [19] gave a discussion of the stability of continuous numerical methods for a special class of nonlinear neutral delay differential equations. In particular, Jackiewicz [2022] systematically investigated the convergence of various numerical methods for more general neutral functional differential equations (NFDEs). In 2009, Yang et al. gave a novel robust stability criteria for stochastic Hopfield neural networks with time delays in [23]. Yang et al. [24] studied the exponential stability on stochastic neural networks with discrete interval and distributed delays in 2010. In 2011, Liu [25] gave the robust stability for Neutral time-varying delay systems with non-linear peturbations. On the stability, Tanikawa studied the values of random zero-sum games in
[26], and in [27] Basin and Calderon-Alyarez gave the delaydependent stability studies for vector nonlinear stochastic systems with multiple delays.

However, these important convergence results are based on the classical Lipschitz conditions. The studies focusing on the stability and convergence of the numerical method for nonlinear NDDEs based on a one-sided Lipschitz condition have not yet been seen in literature until now. By means of a one-sided Lipschitz condition, in the present paper we discuss the stability and convergence of two-step RungeKutta (TSRK) methods for nonlinear NDDEs. Thanks to the one-sided nature of the Lipschitz condition, the error bounds obtained in the present paper are sharper than those given in the references mentioned.

\section{Two-Step Runge-Kutta Methods for NDDEs}

It is the purpose of this paper to investigate the nonlinear stability and convergence properties of the following NDDEs:

$$
\begin{gathered}
y^{\prime}(t)=f\left(t, y(t), y(t-\tau), y^{\prime}(t-\tau)\right), \quad t \in[0, T], \\
y(t)=\varphi(t), \quad t \in[-\tau, 0],
\end{gathered}
$$


where $f:[0, T] \times C^{N} \times C^{N} \times C^{N} \rightarrow C^{N}$ is a given mapping, $\tau$ is a positive delay term, and $\varphi:[-\tau, 0] \rightarrow C^{N}$ is a continuous function. Moreover, we assume that there exist some inner product $\langle\cdot, \cdot\rangle$ and the induced norm $\|\cdot\|$ in $C^{N}$, such that

$$
\begin{gathered}
\operatorname{Re}\left\langle f\left(t, y_{1}, u, v\right)-f\left(t, y_{2}, u, v\right), y_{1}-y_{2}\right\rangle \leq \alpha\left\|y_{1}-y_{2}\right\|^{2}, \\
\left\|f\left(t, y, u_{1}, v\right)-f\left(t, y, u_{2}, v\right)\right\| \leq \beta\left\|u_{1}-u_{2}\right\|, \\
\left\|f\left(t, y, u, v_{1}\right)-f\left(t, y, u, v_{2}\right)\right\| \leq \gamma\left\|v_{1}-v_{2}\right\|, \\
\left\|K\left(t, y, u_{1}, v, w\right)-K\left(t, y, u_{2}, v, w\right)\right\| \leq q\left\|u_{1}-u_{2}\right\|,
\end{gathered}
$$

where $K(t, y, u, v, w)=f(t, y, u, f(t-\tau, u, v, w)), t \in[0, T]$, $\forall y, y_{1}, y_{2}, u, u_{1}, u_{2}, v, v_{1}, v_{2}, w \in C^{N}$, and $\alpha, \beta, \gamma, q$ are constants.

In order to make the error analysis feasible, we always assume that problem (1) has a unique solution $y(t)$ which is sufficiently differentiable and satisfies

$$
\left\|\frac{d^{i} y(t)}{d t^{i}}\right\| \leq M_{i}, \quad i=1,2, \ldots, t \in[-\tau, T]
$$

and denotes the problem class $R(\alpha, \beta, \gamma, q)$ that consists of all NDDEs with (2).

Many numerical methods have been proposed for the numerical solution of problem (1).

In this paper, we are concerned with two-step RungeKutta (TSRK) method of the form

$$
\begin{aligned}
Y_{i}^{(n)}= & y_{n}+h \\
& \times \sum_{j=1}^{s} a_{i j} f\left(t_{n}+c_{j} h, Y_{j}^{(n)}\right), \quad i=1,2, \ldots, s, \\
Y_{i}^{(n-1)}= & y_{n-1}+h \\
& \times \sum_{j=1}^{s} a_{i j} f\left(t_{n-1}+c_{j} h, Y_{j}^{(n-1)}\right), \quad i=1,2, \ldots, s \\
y_{n+1}= & (1-\theta) y_{n}+\theta y_{n-1} \\
& +h \sum_{i=1}^{s} b_{i} f\left(t_{n}+c_{i} h, Y_{i}^{(n)}\right) \\
& +h \sum_{i=1}^{s} \widetilde{b}_{i} f\left(t_{n-1}+c_{i} h, Y_{i}^{(n-1)}\right)
\end{aligned}
$$

where $\sum_{i=1}^{s} b_{i}+\sum_{i=1}^{s} \widetilde{b}_{i}=1+\theta, c_{i}=\sum_{j=1}^{s} a_{i j}, y_{n}$ is the numerical approximation at $t_{n}=n h$ to the analytic solution $y\left(t_{n}\right), h>0$ is a step size, and $0 \leq \theta \leq 1$. The above methods are studied in
[11]. Now we consider the adaptation of the two-step RungeKutta method to (1):

$$
\begin{aligned}
& Y_{i}^{(n)}= y_{n}+h \\
& \times \sum_{j=1}^{s} a_{i j} f\left(t_{n}+c_{j} h, Y_{j}^{(n)}, \bar{Y}_{j}^{(n)}, \widetilde{Y}_{j}^{(n)}\right), \quad i=1,2, \ldots, s \\
& Y_{i}^{(n-1)}= y_{n-1}+h \\
& \times \sum_{j=1}^{s} a_{i j} f\left(t_{n-1}+c_{j} h, Y_{j}^{(n-1)},\right. \\
&\left.\quad \bar{Y}_{j}^{(n-1)}, \widetilde{Y}_{j}^{(n-1)}\right), \quad i=1,2, \ldots, s \\
& y_{n+1}=(1-\theta) y_{n}+\theta y_{n-1} \\
&+h \sum_{i=1}^{s} b_{i} f\left(t_{n}+c_{i} h, Y_{i}^{(n)}, \bar{Y}_{i}^{(n)}, \widetilde{Y}_{i}^{(n)}\right) \\
&+h \sum_{i=1}^{s} \widetilde{b}_{i} f\left(t_{n-1}+c_{i} h, Y_{i}^{(n-1)}, \bar{Y}_{i}^{(n-1)}, \widetilde{Y}_{i}^{(n-1)}\right)
\end{aligned}
$$

where $y_{n}$ is the numerical approximation to the analytic solution $y\left(t_{n}\right)$ with $t_{n}=n h$.

In particular, $y_{0}=\varphi(0)$. The argument $\bar{Y}_{j}^{(n)}$ denotes an approximation to $y\left(t_{n}+c_{j} h-\tau\right)$ and the argument $\widetilde{Y}_{j}^{(n)}$ denotes an approximation to $y^{\prime}\left(t_{n}+c_{j} h-\tau\right)$ which are obtained by a specific interpolation procedure at the point $t=t_{n}+c_{j} h-\tau$. Using values $Y_{j}^{(k)}=\varphi\left(t_{k}+c_{j} h\right)$ with $k<1, t_{k}+c_{j} h \leq 0$.

Let $\tau=(m-\delta) h$ with integer $m$ and $\delta \in[0,1), v, \mu \geq 0$ be integers. Define

$$
\bar{Y}_{j}^{(n)}= \begin{cases}\varphi\left(t_{n}+c_{j} h-\tau\right) & t_{n}+c_{j} h-\tau \leq 0, \\ \sum_{i=-\mu}^{v} \widetilde{L}_{i}(\delta) Y_{j}^{(n-m+i)} & t_{n}+c_{j} h-\tau>0, v+1 \leq m,\end{cases}
$$

where

$$
\begin{gathered}
\widetilde{L}_{i}(\delta)=\prod_{\substack{k=-\mu \\
k \neq i}}^{v}\left(\frac{\delta-k}{i-k}\right), \quad \delta \in[0,1), \\
\widetilde{Y}_{j}^{(n)}=f\left(t_{n}+c_{j} h-\tau, \bar{Y}_{j}^{(n)}, \bar{Y}_{j}^{(n+1)}, \widetilde{Y}_{j}^{(n+1)}\right) .
\end{gathered}
$$

We assume $m \geq v+1$ is to guarantee that no (unknown) values $Y_{j}^{(i)}$ with $i \geq n$ are used in the interpolation procedure.

It should be pointed out that the adopted interpolation procedures (6) is only a class of interpolation procedure for $\bar{Y}_{j}^{(n)}$; there also exist some other types of interpolation procedures, such as numerical schemes which use Hermite interpolation between grid points (see [28-30]). It is the aim 
of our future research to investigate the future adaptation of two-step Runge-Kutta methods to NDDEs by means of other interpolation procedures.

\section{The Nonlinear Stability Analysis}

In this section, we will investigate the stability of the two-step Runge-Kutta methods for NDDEs.

In order to consider the stability property, we also need to consider the perturbed problem of (1):

$$
\begin{gathered}
z^{\prime}(t)=f\left(t, z(t), z(t-\tau), z^{\prime}(t-\tau)\right), \quad t \in[0, T], \\
z(t)=\psi(t), \quad t \in[-\tau, 0],
\end{gathered}
$$

where $\psi:[-\tau, 0] \rightarrow C^{N}$ is a continuous function. The unique exact solution of the problem (8) is denoted as $z(t)$.

Applying the two-step Runge-Kutta method (4a), (4b), and (4c) to (8) leads to

$$
\begin{aligned}
Z_{i}^{(n)}= & z_{n}+h \\
& \times \sum_{j=1}^{s} a_{i j} f\left(t_{n}+c_{j} h, Z_{j}^{(n)}, \bar{Z}_{j}^{(n)}, \widetilde{Z}_{j}^{(n)}\right), \\
& i=1,2, \ldots, s, \\
Z_{i}^{(n-1)}= & z_{n-1}+h \quad i=1,2, \ldots, s, \\
& \times \sum_{j=1}^{s} a_{i j} f\left(t_{n-1}+c_{j} h, Z_{j}^{(n-1)}, \bar{Z}_{j}^{(n-1)}, \widetilde{Z}_{j}^{(n-1)}\right), \\
z_{n+1}= & (1-\theta) z_{n}+\theta z_{n-1} \\
& +h \sum_{i=1}^{s} b_{i} f\left(t_{n}+c_{i} h, Z_{i}^{(n)}, \bar{Z}_{i}^{(n)}, \widetilde{Z}_{i}^{(n)}\right) \\
& +h \sum_{i=1}^{s} \widetilde{b}_{i} f\left(t_{n-1}+c_{i} h, Z_{i}^{(n-1)}, \bar{Z}_{i}^{(n-1)}, \widetilde{Z}_{i}^{(n-1)}\right) .
\end{aligned}
$$

\subsection{Some Concepts}

Definition 1. Let $l$ be a real constant, a two-step Runge-Kutta method with an interpolation procedure is said to be $R(l)$ stable if there exists a constant $C$ dependent only on the method and $l$ such that $(\alpha+\beta+\gamma q) h \leq l$ and

$$
\begin{aligned}
& \left\|y_{n}-z_{n}\right\|^{2} \\
& \quad \leq[2+C(\beta+\gamma q)] \max _{t \leq 0}\|\varphi(t)-\psi(t)\|^{2}, \quad n \geq 1,
\end{aligned}
$$

with step size $h$ satisfying $h m=\tau$, where $m$ is a positive integer.
$\mathrm{GR}(l)$-stability is defined by dropping the restriction $h m=\tau$.

Definition 2. Let $l$ be a real constant, a two-step Runge-Kutta method with an interpolation procedure is said to be $\operatorname{AR}(l)$ stable if

$$
\lim _{n \rightarrow \infty}\left\|y_{n}-z_{n}\right\|=0
$$

with step size $h$ satisfying $(\alpha+\beta+\gamma q) h \leq l$ and $h m=\tau$, where $m$ is a positive integer.

$\operatorname{GAR}(l)$-stability is defined by dropping the restriction $h m=\tau$.

Definition 3. Let $l$ be a real constant; a two-step RungeKutta method with an interpolation procedure is said to be weak $\operatorname{AR}(l)$-stable if, under the conditions of Definition 2, (11) holds when $f$ further satisfies

$$
\left\|f\left(t, u_{1}, v, w\right)-f\left(t, u_{2}, v, w\right)\right\| \leq L\left\|u_{1}-u_{2}\right\|^{p_{0}}
$$

with $p_{0}$ being a positive real number and $L$ being a nonnegative real number.

Weak GAR $(l)$-stability is defined by dropping the restriction $h m=\tau$.

3.2. The Stability of TSRK Methods. Let $w_{n}=y_{n}-z_{n}, W_{i}^{(n)}=$ $Y_{i}^{(n)}-Z_{i}^{(n)}, \bar{W}_{i}^{(n)}=\bar{Y}_{i}^{(n)}-\bar{Z}_{i}^{(n)}, \widetilde{W}_{i}^{(n)}=\widetilde{Y}_{i}^{(n)}-\widetilde{Z}_{i}^{(n)}, Q_{i}^{(n)}=$ $h\left[f\left(t_{n}+c_{i} h, Y_{i}^{(n)}, \bar{Y}_{i}^{(n)}, \widetilde{Y}_{i}^{(n)}\right)-f\left(t_{n}+c_{i} h, Z_{i}^{(n)}, \bar{Z}_{i}^{(n)}, \widetilde{Z}_{i}^{(n)}\right)\right]$, and $i=1,2, \ldots, s$.

It follows from (5a), (5b), (5c), (9a), (9b), and (9c) that

$$
\begin{gathered}
W_{i}^{(n)}=w_{n}+\sum_{j=1}^{s} a_{i j} Q_{j}^{(n)}, \quad i=1,2, \ldots, s, \\
W_{i}^{(n-1)}=w_{n-1}+\sum_{j=1}^{s} a_{i j} Q_{j}^{(n-1)}, \quad i=1,2, \cdots, s, \\
w_{n+1}=(1-\theta) w_{n}+\theta w_{n-1}+\sum_{i=1}^{s} b_{i} Q_{i}^{(n)}+\sum_{i=1}^{s} \widetilde{b}_{i} Q_{i}^{(n-1)} .
\end{gathered}
$$

Now we will write the s-stage TSRK methods (4a), (4b), and $(4 \mathrm{c})$ as a general linear method.

Let $V_{i}^{(n)}=\left(W_{i}^{(n)}, W_{i}^{(n-1)}\right)^{T}$ be the internal stages, $\mu_{n+1}=$ $\left(w_{n+1}, w_{n}\right)^{T}$ the external vectors, and $P_{i}^{(n)}=\left(Q_{i}^{(n)}, Q_{i}^{(n-1)}\right)^{T}$. Then we have a $2(s+1)$-stage partitioned general linear method:

$$
\begin{aligned}
& V_{i}^{(n)}=\sum_{j=1}^{s} C_{i j}^{11} P_{i}^{(n)}+\sum_{j=1}^{s} C_{i j}^{12} \mu_{n}, \quad i=1,2, \ldots, s, \\
& \mu_{n+1}=\sum_{j=1}^{s} C_{i j}^{21} P_{i}^{(n)}+\sum_{j=1}^{s} C_{i j}^{22} \mu_{n}, \quad i=1,2, \ldots, s,
\end{aligned}
$$


where

$$
\begin{gathered}
C_{11}=\left(C_{i j}^{11}\right)=\left(\begin{array}{ll}
A & 0 \\
0 & A
\end{array}\right), \quad C_{12}=\left(C_{i j}^{12}\right)=\left(\begin{array}{ll}
e & 0 \\
0 & e
\end{array}\right), \\
C_{21}=\left(C_{i j}^{21}\right)=\left(\begin{array}{ll}
b & \widetilde{b} \\
0 & 0
\end{array}\right), \quad C_{22}=\left(C_{i j}^{22}\right)=\left(\begin{array}{cc}
1-\theta & \theta \\
0 & 0
\end{array}\right),
\end{gathered}
$$

$$
\begin{gathered}
A=\left(a_{i j}\right), \quad e=(1,1, \ldots 1), \\
b=\left(b_{1}, b_{2}, \ldots, b_{s}\right), \quad \tilde{b}=\left(\widetilde{b}_{1}, \widetilde{b}_{2}, \ldots, \widetilde{b}_{s}\right) .
\end{gathered}
$$

Definition 4. Let $k, l$ be real constants; a TSRK method is said to be $(k, l)$-algebraically stable if there exists a diagonal nonnegative matrix $G$ and $D=\operatorname{diag}\left(d_{1}, d_{2}, \ldots, d_{2 s}\right)$ such that $M=\left(m_{i j}\right)$ is nonnegative, where
In this paper, we use the linear interpolation procedure. Let $\tau=(m-\delta) h$ with integer $m \geq 1$ and $\delta \in[0,1)$.

Define

$$
\begin{gathered}
\bar{Y}_{j}^{(n)}=\delta Y_{j}^{(n-m+1)}+(1-\delta) Y_{j}^{(n-m)}, \\
\bar{Z}_{j}^{(n)}=\delta Z_{j}^{(n-m+1)}+(1-\delta) Z_{j}^{(n-m)}, \quad j=1,2, \ldots, s,
\end{gathered}
$$

where $Y_{j}^{(i)}=\varphi\left(t_{i}+c_{j} h\right)$ and $Z_{j}^{(i)}=\psi\left(t_{i}+c_{j} h\right)$ for $i<0$. When the step size $h$ satisfies $\tau=m h$, we have

$$
\bar{Y}_{j}^{(n)}=Y_{j}^{(n-m)}, \quad \bar{Z}_{j}^{(n)}=Z_{j}^{(n-m)}, \quad j=1,2, \ldots, s .
$$

Theorem 5. Assume that a TSRK method is $(k, l)$-algebraically stable. Then

$$
\begin{aligned}
& \left\|\mu_{n+1}\right\|^{2} \\
& \leq k\left\|\mu_{n}\right\|^{2} \\
& +\sum_{j=1}^{2 s} d_{j}\left[((2 \alpha+\beta+\gamma q) h-2 l)\left\|V_{j}^{(n)}\right\|^{2}+h(\beta+\gamma q)\right. \\
& \left.\quad \times\left(\delta\left\|V_{j}^{(n-m)}\right\|^{2}+(1-\delta)\left\|V_{j}^{(n-m-1)}\right\|^{2}\right)\right] .
\end{aligned}
$$

Proof. It is well known that

$$
\begin{aligned}
\left\|\mu_{n+1}\right\|^{2}-k\left\|\mu_{n}\right\|^{2}-2 \sum_{j=1}^{2 s} d_{j} \operatorname{Re}\left\langle V_{j}^{(n)}, P_{j}^{(n)}-l V_{j}^{(n)}\right\rangle \\
=-\sum_{i=1}^{2 s+2} \sum_{j=1}^{2 s+2} M_{i j}\left\langle r_{i}, r_{j}\right\rangle,
\end{aligned}
$$

where

$$
\begin{gathered}
r_{1}=w_{n+1}, \quad r_{2}=w_{n}, \\
r_{j}=Q_{j-2}^{(n)}, \quad j=3,4, \ldots, s+2, \\
r_{j}=Q_{j-s-2}^{(n-1)}, \quad j=s+3, s+4, \ldots, 2 s+2 .
\end{gathered}
$$

By means of $(k, l)$-algebraical stability of the method, we have

$$
\left\|\mu_{n+1}\right\|^{2} \leq k\left\|\mu_{n}\right\|^{2}+2 \sum_{j=1}^{2 s} d_{j} \operatorname{Re}\left\langle V_{j}^{(n)}, P_{j}^{(n)}-l V_{j}^{(n)}\right\rangle .
$$

It follows from (2) and (6) that

$$
\begin{gathered}
2 \operatorname{Re}\left\langle W_{j}^{(n)}, Q_{j}^{(n)}\right\rangle \\
=2 h \operatorname{Re}\left\langle W_{j}^{(n)}, f\left(t_{n}+c_{j} h, Y_{j}^{(n)}, \bar{Y}_{j}^{(n)}, \widetilde{Y}_{j}^{(n)}\right)\right. \\
\left.-f\left(t_{n}+c_{j} h, Z_{j}^{(n)}, \bar{Y}_{j}^{(n)}, \widetilde{Y}_{j}^{(n)}\right)\right\rangle \\
+2 h \operatorname{Re}\left\langle W_{j}^{(n)}, f\left(t_{n}+c_{j} h, Z_{j}^{(n)}, \bar{Y}_{j}^{(n)}, \widetilde{Y}_{j}^{(n)}\right)\right. \\
\left.-f\left(t_{n}+c_{j} h, Z_{j}^{(n)}, \bar{Z}_{j}^{(n)}, \widetilde{Y}_{j}^{(n)}\right)\right\rangle \\
+2 h \operatorname{Re}\left\langle W_{j}^{(n)}, f\left(t_{n}+c_{j} h, Z_{j}^{(n)}, \bar{Z}_{j}^{(n)}, \widetilde{Y}_{j}^{(n)}\right)\right. \\
\left.\quad-f\left(t_{n}+c_{j} h, Z_{j}^{(n)}, \bar{Z}_{j}^{(n)}, \widetilde{Z}_{j}^{(n)}\right)\right\rangle \\
\leq 2 h \alpha\left\|W_{j}^{(n)}\right\|^{2}+2 h \beta\left\|W_{j}^{(n)}\right\| \\
\cdot\left\|\bar{W}_{j}^{(n)}\right\|+2 h \gamma\left\|W_{j}^{(n)}\right\| \cdot\left\|\widetilde{W}_{j}^{(n)}\right\| \\
\leq 2 h \alpha\left\|W_{j}^{(n)}\right\|^{2}+2 h \beta\left\|W_{j}^{(n)}\right\| \\
\cdot\left\|\bar{W}_{j}^{(n)}\right\|+2 h \gamma q\left\|W_{j}^{(n)}\right\| \cdot\left\|\bar{W}_{j}^{(n)}\right\| \\
\leq 2 h \alpha\left\|W_{j}^{(n)}\right\|^{2}+2 h(\beta+\gamma q)\left\|W_{j}^{(n)}\right\| \cdot\left\|\bar{W}_{j}^{(n)}\right\| \\
\leq 2 h \alpha\left\|W_{j}^{(n)}\right\|^{2}+2 h(\beta+\gamma q)\left\|W_{j}^{(n)}\right\| \\
\cdot\left(\delta\left\|W_{j}^{(n-m)}\right\|+(1-\delta)\left\|W_{j}^{(n-1-m)}\right\|\right)
\end{gathered}
$$




$$
\begin{aligned}
\leq & h(2 \alpha+\beta+\gamma q)\left\|W_{j}^{(n)}\right\|^{2} \\
& +h(\beta+\gamma q)\left(\delta\left\|W_{j}^{(n-m)}\right\|^{2}+(1-\delta)\left\|W_{j}^{(n-1-m)}\right\|^{2}\right) .
\end{aligned}
$$

Substitution into (20) gives (19).

Theorem 6. Assume that a TSRK method is $(k, l)$-algebraically stable and $k \leq 1$. Then the method with linear interpolation procedure is $\mathrm{GR}(\mathrm{l})$-stable.

Proof. The inequality $(\alpha+\beta+\gamma q) h \leq l$ and Theorem 5 lead to

$$
\begin{aligned}
&\left\|\mu_{n+1}\right\|^{2} \leq\left\|\mu_{n}\right\|^{2} \\
&+\sum_{j=1}^{2 s} d_{j}\left[-(\beta+\gamma q) h\left\|V_{j}^{(n)}\right\|^{2}+h(\beta+\gamma q)\right. \\
&\left.\quad \times\left(\delta\left\|V_{j}^{(n-m)}\right\|^{2}+(1-\delta)\left\|V_{j}^{(n-m-1)}\right\|^{2}\right)\right] .
\end{aligned}
$$

By induction, we have

$$
\begin{aligned}
& \left\|\mu_{n+1}\right\|^{2} \\
& \leq\left\|\mu_{0}\right\|^{2} \\
& \quad+h \sum_{j=1}^{2 s} d_{j}(\beta+\gamma q)\left(\delta \sum_{i=-m}^{-1}\left\|V_{j}^{(i)}\right\|^{2}\right. \\
& \left.\quad+(1-\delta) \sum_{i=-m-1}^{-1}\left\|V_{j}^{(i)}\right\|^{2}\right) \\
& \leq\left\|\mu_{0}\right\|^{2}+h \sum_{j=1}^{2 s} d_{j}(\beta+\gamma q) \max _{-m-1 \leq i \leq-1}\left\|V_{j}^{(i)}\right\|^{2} \\
& \quad \times\left[\delta(m-1)+(1-\delta) m_{m}\left\|^{2}+(\beta+\gamma q) \tau \sum_{j=1}^{2 s} d_{j} \max _{-m-1 \leq i \leq-1}\right\| V_{j}^{(i)} \|^{2}\right. \\
& \leq \quad\left\|\mu_{0}\right\|^{2}+(\beta+\gamma q) \tau \sum_{j=1}^{2 s} d_{j} \max _{t \leq 0}\|\varphi(t)-\psi(t)\|^{2} .
\end{aligned}
$$

Because $\mu_{n+1}=\left(w_{n+1}, w_{n}\right)^{T}$, so we have

$$
\begin{aligned}
\left\|w_{n}\right\|^{2} \leq & \left\|w_{0}\right\|^{2} \\
& +\left\|w_{-1}\right\|^{2}+(\beta+\gamma q) \tau \\
& \times \sum_{j=1}^{2 s} d_{j} \max _{t \leq 0}\|\varphi(t)-\psi(t)\|^{2} \\
\leq & 2 \max _{t \leq 0}\|\varphi(t)-\psi(t)\|^{2}+(\beta+\gamma q) \tau
\end{aligned}
$$

$$
\begin{aligned}
& \times \sum_{j=1}^{2 s} d_{j} \max _{t \leq 0}\|\varphi(t)-\psi(t)\|^{2} \\
\leq & {[2+C(\beta+\gamma q) \tau] \max _{t \leq 0}\|\varphi(t)-\psi(t)\|^{2} . }
\end{aligned}
$$

Therefore, it is $\operatorname{GR}(l)$-stable, where $C=\sum_{j=1}^{2 s} d_{j}$.

Theorem 7. Assume that a TSRK method is $(k, l)$-algebraically stable and $k<1$. Then the method with linear interpolation procedure is $\mathrm{GAR}(\mathrm{l})$-stable.

Proof. Let $\mu=(2 \alpha+\beta+\gamma q) h-2 l$ and $\bar{k}=\max \{k,(\beta+$ $\left.\gamma q) h /(-\mu)^{1 /(m+1)}\right\}$.

Then, when $(\alpha+\beta+\gamma q) h<l$, we have $\mu<-(\beta+\gamma q) h$ and $0<\bar{k}<1$.

The application of Theorem 5 yields

$$
\begin{aligned}
& \left\|\mu_{n+1}\right\|^{2} \leq \bar{k}\left\|\mu_{n}\right\|^{2} \\
& +\sum_{j=1}^{2 s} d_{j}\left[\mu\left\|V_{j}^{(n)}\right\|^{2}+h(\beta+\gamma q)\right. \\
& \left.\quad \times\left(\delta\left\|V_{j}^{(n-m)}\right\|^{2}+(1-\delta)\left\|V_{j}^{(n-m-1)}\right\|^{2}\right)\right] .
\end{aligned}
$$

By induction, we have

$$
\begin{gathered}
\left\|\mu_{n+1}\right\|^{2} \leq \bar{k}^{n+1}\left\|\mu_{0}\right\|^{2} \\
+\sum_{i=0}^{n} \bar{k}^{n-i} \sum_{j=1}^{2 s} d_{j}\left[\mu\left\|V_{j}^{(i)}\right\|^{2}+h(\beta+\gamma q)\right. \\
\times\left(\delta\left\|V_{j}^{(i-m)}\right\|^{2}+(1-\delta)\right. \\
\left.\left.\times\left\|V_{j}^{(i-m-1)}\right\|^{2}\right)\right] \\
\leq \bar{k}^{n+1}\left\|\mu_{0}\right\|^{2} \sum_{j=1}^{2 s} d_{j} \sum_{i=0}^{n-m}\left(\bar{k}^{n-i} \mu+h(\beta+\gamma q) \delta \bar{k}^{n-m-i}\right. \\
\left.+h(\beta+\gamma q)(1-\delta) \bar{k}^{n-m-i-1}\right) \\
\times\left\|V_{j}^{(i)}\right\|^{2}+h(\beta+\gamma q) \\
\times\left(\begin{array}{l}
\delta \sum_{i=-m}^{-1} \bar{k}^{n-m-i}\left\|V_{j}^{(i)}\right\|^{2} \\
\left.+(1-\delta) \sum_{i=-m-1}^{-1} \bar{k}^{n-m-i-1}\left\|V_{j}^{(i)}\right\|^{2}\right)
\end{array}\right]
\end{gathered}
$$




$$
\begin{aligned}
& \leq \bar{k}^{n+1}\left\|\mu_{0}\right\|^{2} \\
&+\sum_{j=1}^{2 s} d_{j}\left[\sum _ { i = 0 } ^ { n - m } \overline { k } ^ { n - m - i - 1 } \left(\bar{k}^{m+1} \mu+h(\beta+\gamma q) \delta \bar{k}\right.\right. \\
&+h(\beta+\gamma q)(1-\delta))\left\|V_{j}^{(i)}\right\|^{2} \\
&\left.+\bar{k}^{n-m} h(\beta+\gamma q) \sum_{i=-m-1}^{-1}\left\|V_{j}^{(i)}\right\|^{2}\right] .
\end{aligned}
$$

On the other hand,

$$
\begin{aligned}
\mu \bar{k}^{m+1} & +h \bar{k}(\beta+\gamma q) \delta \\
& +h(\beta+\gamma q)(1-\delta) \\
\leq & h(\beta+\gamma q)+h \bar{k}(\beta+\gamma q) \delta \\
& +h(\beta+\gamma q)(1-\delta) \leq 0 .
\end{aligned}
$$

Considering $d_{j} \geq 0, j=1,2, \ldots, s$ and $0<\bar{k}<1$, we have

$$
\lim _{n \rightarrow \infty}\left\|\mu_{n}\right\|=0
$$

Because $\mu_{n+1}=\left(w_{n+1}, w_{n}\right)^{T}$, so we have

$$
\lim _{n \rightarrow \infty}\left\|w_{n}\right\|=0
$$

which shows that the method is $\operatorname{GAR}(l)$-stable.

Theorem 8. Assume that a TSRK method is $(k, l)$-algebraically stable, $k<1$, and $d_{j}>0, j=1,2 \ldots, s$. Then the method with linear interpolation procedure is weak GAR(l)-stable.

Proof. It follows from Theorem 5 that

$$
\begin{aligned}
\left\|\mu_{n+1}\right\|^{2} \leq & \left\|\mu_{n}\right\|^{2}+\sum_{j=1}^{2 s} d_{j} \\
\times & -\sigma\left\|V_{j}^{(n)}\right\|^{2}+h(\beta+\gamma q) \\
& \left.\times\left(\delta\left\|V_{j}^{(n-m)}\right\|^{2}+(1-\delta)\left\|V_{j}^{(n-m-1)}\right\|^{2}\right)\right],
\end{aligned}
$$

where $\sigma=2 l-(2 \alpha+\beta+\gamma q) h$. When $(\alpha+\beta+\gamma q) h<l$, we have $\sigma>0$. Analogous to Theorem 6 , we can easily obtain

$$
\begin{gathered}
\left\|\mu_{n+1}\right\|^{2} \leq\left\|\mu_{0}\right\|^{2} \\
+\sum_{j=1}^{2 s} d_{j}\left[(\beta+\gamma q) \tau \max _{-m-1 \leq i \leq-1}\left\|V_{j}^{(i)}\right\|^{2}\right. \\
\left.-\sigma \sum_{i=1}^{n}\left\|V_{j}^{(i)}\right\|^{2}\right]
\end{gathered}
$$

which shows

$$
\begin{array}{ll}
\lim _{n \rightarrow \infty} \sum_{j=1}^{2 s} d_{j}\left\|V_{j}^{(n)}\right\|^{2}=0, & \lim _{n \rightarrow \infty} \sum_{j=1}^{2 s} d_{j}\left\|W_{j}^{(n)}\right\|^{2}=0, \\
\lim _{n \rightarrow \infty} \sum_{j=1}^{2 s} d_{j}\left\|\bar{V}_{j}^{(n)}\right\|^{2}=0, & \lim _{n \rightarrow \infty} \sum_{j=1}^{2 s} d_{j}\left\|\bar{W}_{j}^{(n)}\right\|^{2}=0 .
\end{array}
$$

On the other hand,

$$
\begin{aligned}
\left\|Q_{i}^{(n)}\right\| \leq h \| f\left(t_{n}+c_{i} h, Y_{i}^{(n)}, \bar{Y}_{i}^{(n)}, \widetilde{Y}_{i}^{(n)}\right) & -f\left(t_{n}+c_{i} h, Z_{i}^{(n)}, \bar{Y}_{i}^{(n)}, \widetilde{Y}_{i}^{(n)}\right) \| \\
+ & h \| f\left(t_{n}+c_{i} h, Z_{i}^{(n)}, \bar{Y}_{i}^{(n)}, \widetilde{Y}_{i}^{(n)}\right) \\
& -f\left(t_{n}+c_{i} h, Z_{i}^{(n)}, \bar{Z}_{i}^{(n)}, \widetilde{Z}_{i}^{(n)}\right) \| \\
+ & h \| f\left(t_{n}+c_{i} h, Z_{i}^{(n)}, \bar{Z}_{i}^{(n)}, \widetilde{Y}_{i}^{(n)}\right) \\
& -f\left(t_{n}+c_{i} h, Z_{i}^{(n)}, \bar{Z}_{i}^{(n)}, \widetilde{Z}_{i}^{(n)}\right) \| \\
\leq & h \| f\left(t_{n}+c_{i} h, Y_{i}^{(n)}, \bar{Y}_{i}^{(n)}, \widetilde{Y}_{i}^{(n)}\right) \\
& -f\left(t_{n}+c_{i} h, Z_{i}^{(n)}, \bar{Y}_{i}^{(n)}, \widetilde{Y}_{i}^{(n)}\right) \| \\
+ & h \beta\left\|\bar{W}_{j}^{(n)}\right\|+h \gamma\left\|\widetilde{W}_{j}^{(n)}\right\| \\
+ & h \beta\left\|\bar{W}_{j}^{(n)}\right\|+h \gamma q\left\|\bar{W}_{j}^{(n)}\right\| . \\
& -f\left(t_{n}+c_{i} h, Y_{i}^{(n)}, \bar{Y}_{i}^{(n)}, \widetilde{Y}_{i}^{(n)}\right) \\
& \\
+ &
\end{aligned}
$$

In view of (12) and (35), we have

$$
\lim _{n \rightarrow \infty} \sum_{j=1}^{2 s} d_{j}\left\|Q_{j}^{(n)}\right\|^{2}=0
$$

Considering (9a), (9b), (9c), (22), and (37) with $d_{j}>0$, we have

$$
\lim _{n \rightarrow \infty}\left\|\mu_{n}\right\|=0
$$

Because $\mu_{n+1}=\left(w_{n+1}, w_{n}\right)^{T}$, so we have

$$
\lim _{n \rightarrow \infty}\left\|w_{n}\right\|=0
$$

which shows that the method is weak $\operatorname{GAR}(l)$-stable. 


\section{The Convergence of TSRK Method for NDDEs}

4.1. Some Concepts. In order to study the convergence of the method, we define

$$
\begin{aligned}
Y^{(n)}= & \left(Y_{1}^{(n)^{T}}, \ldots, Y_{s}^{(n)^{T}}, Y_{1}^{(n-1)^{T}}, \ldots, Y_{s}^{(n-1)^{T}}\right)^{T} \in C^{2 s N}, \\
\bar{Y}^{(n)}= & \left(\bar{Y}_{1}^{(n)^{T}}, \ldots, \bar{Y}_{s}^{(n)^{T}}, \bar{Y}_{1}^{(n-1)^{T}}, \ldots, \bar{Y}_{s}^{(n-1)^{T}}\right)^{T} \in C^{2 s N}, \\
\tilde{Y}^{(n)}= & \left(\widetilde{Y}_{1}^{(n)^{T}}, \ldots, \tilde{Y}_{s}^{(n)^{T}}, \widetilde{Y}_{1}^{(n-1)^{T}}, \ldots, \widetilde{Y}_{s}^{(n-1)^{T}}\right)^{T} \in C^{2 s N}, \\
F\left(t_{n}, Y^{(n)}, \bar{Y}^{(n)}, \tilde{Y}^{(n)}\right) & \left(y^{(n+1)^{T}}, y^{(n)^{T}}\right)^{T} \in C^{2 N}, \\
= & \left(f\left(t_{n}+c_{1} h, Y_{1}^{(n)}, \bar{Y}_{1}^{(n)}, \tilde{Y}_{1}^{(n)}\right)^{T}, \ldots,\right. \\
& f\left(t_{n}+c_{s} h, Y_{s}^{(n)}, \bar{Y}_{s}^{(n)}, \tilde{Y}_{s}^{(n)}\right)^{T}, \\
& f\left(t_{n-1}+c_{1} h, Y_{1}^{(n-1)}, \bar{Y}_{1}^{(n-1)}, \widetilde{Y}_{1}^{(n-1)}\right)^{T}, \ldots, \\
& \left.f\left(t_{n-1}+c_{s} h, Y_{s}^{(n-1)}, \bar{Y}_{s}^{(n-1)}, \widetilde{Y}_{s}^{(n-1)}\right)^{T}\right)^{T} \in C^{2 s N}
\end{aligned}
$$

Thus, process (5a), (5b), and (5c) can be written in the more compact form

$$
\begin{aligned}
& Y^{(n)}=h C_{11} F\left(t_{n}, Y^{(n)}, \bar{Y}^{(n)}, \tilde{Y}^{(n)}\right)+C_{12} \zeta^{(n)}, \\
& \zeta^{(n+1)}=h C_{21} F\left(t_{n}, Y^{(n)}, \bar{Y}^{(n)}, \widetilde{Y}^{(n)}\right)+C_{22} \zeta^{(n)} .
\end{aligned}
$$

Definition 9. Method (4a), (4b), and (4c) with an interpolation procedure is said to be $D$-convergent of order $p$ if the global error satisfies a bound of the form

$$
\begin{array}{r}
\left\|H\left(t_{n}\right)-y^{(n)}\right\| \\
\leq \rho_{1}\left(t_{n}\right)\left(\left\|H\left(t_{0}\right)-y^{(0)}\right\|+\max _{k \leq 0}\left\|Y\left(t_{k}\right)-y^{(k)}\right\|+h^{p}\right), \\
n \geq 1, h \in\left(0, h_{0}\right],
\end{array}
$$

where $H(t)$ is defined by

$$
\begin{gathered}
H(t)=(y(t+h), y(t)) \in C^{2 r N}, \\
Y(t)=\left(y\left(t+c_{1} h\right), \ldots, y\left(t+c_{s} h\right),\right. \\
\left.y\left(t-h+c_{1} h\right), \ldots, y\left(t-h+c_{s} h\right)\right) \in C^{2 s N} .
\end{gathered}
$$

$\rho_{1}(t)$ and $h_{0}$ depend on $M_{i}, \alpha, \beta, \gamma$, and $\tau$.
Definition 10. TSRK method (4a), (4b), and (4c) is said to be algebraically stable if there exist a real symmetric, positive definite $2 r \times 2 r$ matrix $G$ and a nonnegative diagonal $2 s \times 2 s$ matrix $D$ such that the matrix

$$
M=\left[\begin{array}{cc}
G-C_{22}^{T} G C_{22} & C_{12}^{T} D-C_{22}^{T} G C_{21} \\
D C_{12}-C_{21}^{T} G C_{22} & D C_{11}+C_{11}^{T} D-C_{21}^{T} G C_{21}
\end{array}\right]
$$

is nonnegative definite.

Definition 11. TSRK method (4a), (4b), and (4c) is said to be diagonally stable if there exist an $2 s \times 2 s$ diagonal matrix $Q>0$ such that the matrix $Q C_{11}+C_{11}^{T} Q$ is positive definite.

Remark 12 . The concepts of algebraic stability and diagonal stability of TSRK method are the generalizations of corresponding concepts of Runge-Kutta methods. Although it is difficult to examine these conditions, many results have been found; in particular, there exist algebraically stable and diagonally stable multistep formulas of arbitrarily high order (cf. [31]).

Definition 13. TSRK method (4a), (4b), and (4c) is said to have generalized stage order $P$ if $P$ is the largest integer which possesses the following properties.

For any given problem $(1)$ and $h \in\left[0, \bar{h}_{0}\right]$, there exists an abstract function $H^{h}(t)$,

$$
H^{h}(t)=\left(H_{1}^{h}(t), H_{2}^{h}(t)\right) \in C^{2 N},
$$

such that

$$
\begin{gathered}
\left\|H(t)-H^{h}(t)\right\| \leq p_{1} h^{p}, \\
\left\|\Delta^{h}(t)\right\| \leq p_{1} h^{p+1}, \\
\left\|\delta^{h}(t)\right\| \leq p_{1} h^{p+1},
\end{gathered}
$$

where the maximum step size $\bar{h}_{0}>0$ and the constant $p_{1}$ depend only on the method and the bounds $M_{i}, \Delta^{h}(t)$, and $\delta^{h}(t)$; they are defined by;

$$
\begin{gathered}
Y(t)=h C_{11} Y^{\prime}(t)+C_{12} H^{h}(t)+\Delta^{h}(t), \\
H^{h}(t+h)=h C_{21} Y^{\prime}(t)+C_{22} H^{h}(t)+\delta^{h}(t) .
\end{gathered}
$$

The function $Y^{\prime}(t)$ is defined by

$$
\begin{aligned}
Y(t)=( & y^{\prime}\left(t+c_{1} h\right), \ldots, y^{\prime}\left(t+c_{s} h\right), \\
& \left.y^{\prime}\left(t-h+c_{1} h\right), \ldots, y^{\prime}\left(t-h+c_{s} h\right)\right) \in C^{2 s N} .
\end{aligned}
$$

In particular when $H(t)=H^{h}(t)$, generalized stage order is called stage order.

4.2. D-Convergence and Proofs. In this section, we focus on the error analysis of TSRK method for (1). For the sake of simplicity, we always assume that all constants $h_{i}, \gamma_{i}, d_{i}$, and 
$L_{i}$ are dependent only on the method, some of the bounds $M_{i}$, and the parameters $\alpha, \beta, \gamma$, and $\tau$.

First, we give a preliminary result which will later be used several times. For any $Y, \bar{Y}, \widetilde{Y}, Z, \bar{Z}, \widetilde{Z} \in C^{2 s N}$ for $Y^{(n)}, \bar{Y}^{(n)}$, $\widetilde{Y}^{(n)}, Z^{(n)}, \bar{Z}^{(n)}, \widetilde{Z}^{(n)}$, and $\zeta, \omega \in C^{2 N}$ for $\zeta^{(n+1)}, \omega^{(n+1)}$ where $\Phi^{(n+1)}=\left(z^{(n+1)}, z^{(n)}\right)$. Define $\widetilde{\Delta}$ and $\widetilde{\delta}$ by

$$
\begin{aligned}
\widetilde{\Delta} & =Y-Z-h C_{11}(F(t, Y, \bar{Y}, \widetilde{Y})-F(t, Z, \bar{Z}, \widetilde{Z})), \\
\widetilde{\delta} & =\zeta-\omega-h C_{21}(F(t, Y, \bar{Y}, \widetilde{Y})-F(t, Z, \bar{Z}, \widetilde{Z})) .
\end{aligned}
$$

Theorem 14. Suppose that method (4a), (4b), and (4c) is diagonally stable. Then there exist constants $h_{1}, p_{2}$, and $p_{3}$ such that

$$
\begin{array}{r}
\|Y-Z\| \leq p_{2}(\|\widetilde{\Delta}\|+h\|\bar{Y}-\bar{Z}\|+h\|\widetilde{Y}-\widetilde{Z}\|), \quad h \in\left(0, h_{1}\right], \\
\|\zeta-\Phi\| \leq p_{3}(\|\widetilde{\Delta}\|+\|\widetilde{\delta}\|+h\|\bar{Y}-\bar{Z}\|+h\|\widetilde{Y}-\widetilde{Z}\|), \quad \\
h \in\left(0, h_{1}\right] .
\end{array}
$$

Proof. Since the method (4a), (4b), and (4c) is diagonally stable, there exists a positive definite diagonal matrix $Q$ such that the matrix $E=Q C_{11}+C_{11}^{T} Q$ is positive definite. Therefore, the matrix $C_{11}$ is obviously nonsingular, and there exists an $l>0$ which depends only on the method such that the matrix

$$
E_{l}=C_{11}^{-T} E C_{11}-2 l Q
$$

is also positive definite.

Define

$$
\begin{gathered}
W=Y-Z, \quad \bar{W}=\bar{Y}-\bar{Z}, \quad \widetilde{W}=\widetilde{Y}-\widetilde{Z}, \\
K_{1}=h(F(t, Y, \bar{Y}, \widetilde{Y})-F(t, Z, \bar{Y}, \widetilde{Y})), \\
K_{2}=h(F(t, Z, \bar{Y}, \widetilde{Y})-F(t, Z, \bar{Z}, \widetilde{Y})), \\
K_{3}=h(F(t, Z, \bar{Z}, \widetilde{Y})-F(t, Z, \bar{Z}, \widetilde{Z})) .
\end{gathered}
$$

Then

$$
\begin{gathered}
\widetilde{\Delta}=W-C_{11}\left(K_{1}+K_{2}+K_{3}\right), \\
\widetilde{\delta}=\zeta-\varpi-C_{21}\left(K_{1}+K_{2}+K_{3}\right) .
\end{gathered}
$$

Using (2), (6), (53a), and (53b), we have, for $h \alpha \leq l$,

$$
\begin{aligned}
0 \leq & \langle W, 2 l Q W\rangle+2 \operatorname{Re}\left\langle K_{1},-Q W\right\rangle \\
= & -\left\langle W, E_{l} W\right\rangle+2 \operatorname{Re}\left\langle W, Q\left(C_{11}^{-1}-K_{1}\right)\right\rangle \\
\leq & -\lambda_{l}\|W\|^{2}+2 \operatorname{Re}\left\langle W, Q\left(C_{11}^{-1} \widetilde{\Delta}+K_{2}+K_{3}\right)\right\rangle \\
\leq & -\lambda_{l}\|W\|^{2}+2\left\|Q C_{11}^{-1}\right\|\|W\|\|\widetilde{\Delta}\| \\
& +2 h \beta\|Q\|\|W\|\|\bar{W}\|+2 h \gamma\|Q\|\|W\|\|\widetilde{W}\|,
\end{aligned}
$$

where $\lambda_{l}$ is the minimum eigenvalue of $E_{l}$. Therefore,

$$
\|Y-Z\| \leq p_{1}(\|\widetilde{\Delta}\|+h\|\bar{Y}-\bar{Z}\|+h\|\widetilde{Y}-\widetilde{Z}\|), \quad h \in\left(0, h_{1}\right],
$$

where

$$
\begin{gathered}
p_{1}=\frac{2}{\lambda_{l}} \max \left(\left\|Q C_{11}^{-1}\right\|, \beta\|Q\|, \gamma\|Q\|\right), \\
h_{1}= \begin{cases}1 & \alpha \leq 0, \\
\min \left(1, \frac{l}{\alpha}\right) & \alpha>0 .\end{cases}
\end{gathered}
$$

From (50a), (56a), and (56b), it follows that

$$
\begin{aligned}
\|\zeta-\Phi\|= & \left\|\widetilde{\delta}+C_{21} C_{11}^{-1}(W-\widetilde{\Delta})\right\| \\
\leq & \|\widetilde{\delta}\|+p_{1} h\left\|C_{21} C_{11}^{-1}\right\|(\|\bar{W}\|+\|\widetilde{W}\|) \\
& +\left(1+p_{1}\right)\left\|C_{21} C_{11}^{-1}\right\| \cdot\|\widetilde{\Delta}\| \\
\leq & p_{3}(\|\widetilde{\Delta}\|+\|\widetilde{\delta}\|+h\|\bar{Y}-\bar{Z}\|+h\|\widetilde{Y}-\widetilde{Z}\|), \\
& h \in\left(0, h_{1}\right],
\end{aligned}
$$

where $p_{3}=\max \left\{1,\left(1+p_{2}\right)\left\|C_{21} C_{11}^{-1}\right\|\right\}$, which completes the proof of Theorem 14.

Consider the compact form of (9a), (9b), and (9c):

$$
\begin{gathered}
Z^{(n)}=h C_{11} F\left(t_{n}, Z^{(n)}, \bar{Z}^{(n)}, \widetilde{Z}^{(n)}\right)+C_{12} \omega^{(n)}, \\
\omega^{(n+1)}=h C_{21} F\left(t_{n}, Z^{(n)}, \bar{Z}^{(n)}, \widetilde{Z}^{(n)}\right)+C_{22} \omega^{(n)},
\end{gathered}
$$

where

$$
\begin{gathered}
Z^{(n)}=\left(Z_{1}^{(n)}, \ldots, Z_{s}^{(n)}, Z_{1}^{(n-1)}, \ldots, Z_{s}^{(n-1)}\right) \in C^{2 s N}, \\
\bar{Z}^{(n)}=\left(\bar{Z}_{1}^{(n)}, \ldots, \bar{Z}_{s}^{(n)}, \bar{Z}_{1}^{(n-1)}, \ldots, \bar{Z}_{s}^{(n-1)}\right) \in C^{2 s N}, \\
\widetilde{Z}^{(n)}=\left(\widetilde{Z}_{1}^{(n)}, \ldots, \widetilde{Z}_{s}^{(n)}, \widetilde{Z}_{1}^{(n-1)}, \ldots, \widetilde{Z}_{s}^{(n-1)}\right) \in C^{2 s N}, \\
\omega^{(n+1)}=\left(z^{(n+1)}, z^{(n)}\right) \in C^{2 N} .
\end{gathered}
$$

Theorem 15. Suppose that the method (4a), (4b), and (4c) is algebraically stable for the matrices $G$ and $D$. Then for (41a), (41b), (58a), and (58b) we have

$$
\begin{aligned}
\left\|\zeta^{(n+1)}-\Phi^{(n+1)}\right\|_{G}^{2} \leq & \left\|\zeta^{(n)}-\Phi^{(n)}\right\|_{G}^{2} \\
+p_{4} h( & \left\|Y^{(n)}-Z^{(n)}\right\|^{2} \\
& +\left\|Y^{(n-m)}-Z^{(n-m)}\right\|^{2} \\
& \left.+\left\|\widetilde{Y}^{(n)}-\widetilde{Z}^{(n)}\right\|^{2}\right),
\end{aligned}
$$


where $p_{4}=\|D\| \cdot \max \{(2 \alpha+\beta+\gamma), \beta, \gamma\},\|\cdot\|$ is a norm on $C^{2 s N}$ defined by

$$
\begin{gathered}
\|U\|_{G}=\langle U, G U\rangle^{1 / 2}=\left(\sum_{i, j=1}^{2 s} g_{i j}\left\langle u_{i}, u_{j}\right\rangle\right)^{1 / 2}, \\
U=\left(u_{1}, u_{2}, \ldots, u_{2 s}\right) \in C^{2 s N}, \quad u_{i} \in C^{N} .
\end{gathered}
$$

Proof. Define $u^{(n)}=\zeta^{(n)}-\omega^{(n)}$. We get from (52a), (52b), (52c), and (52d) that

$$
\begin{gathered}
K^{(n)}=h\left(F\left(t_{n}, Y^{(n)}, \bar{Y}^{(n)}, \widetilde{Y}^{(n)}\right)-F\left(t_{n}, Z^{(n)}, \bar{Z}^{(n)}, \widetilde{Z}^{(n)}\right)\right), \\
W^{(n)}=C_{11} K^{(n)}+C_{12} u^{(n)}, \\
u^{(n+1)}=C_{21} K^{(n)}+C_{22} u^{(n)} .
\end{gathered}
$$

With algebraic stability, the matrix

$$
M=\left[\begin{array}{cc}
G-C_{22}^{T} G C_{22} & C_{12}^{T} D-C_{22}^{T} G C_{21} \\
D C_{12}-C_{21}^{T} G C_{22} & D C_{11}+C_{11}^{T} D-C_{21}^{T} G C_{21}
\end{array}\right]
$$

is nonnegative definite. As in [32], we have

$$
\begin{array}{r}
\left\langle u^{(n+1)}, G u^{(n+1)}\right\rangle-\left\langle u^{(n)}, G u^{(n)}\right\rangle-2 \operatorname{Re}\left\langle W^{(n)}, D K^{(n)}\right\rangle \\
=-\left\langle\left\langle u^{(n)}, K^{(n)}\right\rangle, M\left\langle u^{(n)}, K^{(n)}\right\rangle\right\rangle \leq 0 .
\end{array}
$$

Using (2), we further obtain

$$
\begin{aligned}
&\left\langle u^{(n+1)}, G u^{(n+1)}\right\rangle \\
& \leq\left\langle u^{(n)}, G u^{(n)}\right\rangle+2 \operatorname{Re}\left\langle W^{(n)}, D K^{(n)}\right\rangle \\
& \leq\left\langle u^{(n)}, G u^{(n)}\right\rangle+2 \operatorname{Re}\left\langle W^{(n)}, D K_{1}^{(n)}\right\rangle \\
&+2 \operatorname{Re}\left\langle W^{(n)}, D K_{2}^{(n)}+D K_{3}^{(n)}\right\rangle \\
& \leq\left\langle u^{(n)}, G u^{(n)}\right\rangle+2 h \alpha\left\langle W^{(n)}, D W^{(n)}\right\rangle \\
&+2 h \beta\left\|D^{1 / 2} W^{(n)}\right\| \cdot\left\|D^{1 / 2} \bar{W}^{(n)}\right\| \\
&+2 h \gamma\left\|D^{1 / 2} W^{(n)}\right\| \cdot\left\|D^{1 / 2} \widetilde{W}^{(n)}\right\| \\
& \leq\left\langle u^{(n)}, G u^{(n)}\right\rangle+2 h \alpha\left\langle W^{(n)}, D W^{(n)}\right\rangle \\
&+h \beta\left(\|D\| \cdot\left\|W^{(n)}\right\|^{2}+\|D\| \cdot\left\|\bar{W}^{(n)}\right\|^{2}\right) \\
&+h \gamma\left(\|D\| \cdot\left\|W^{(n)}\right\|^{2}+\|D\| \cdot\left\|\widetilde{W}^{(n)}\right\|^{2}\right) \\
& \leq\left\langle u^{(n)}, G u^{(n)}\right\rangle+(2 h \alpha+h \beta+h \gamma)\|D\| \cdot\left\|W^{(n)}\right\|^{2} \\
&+\|D\|\left(h \beta\left\|W^{(n-m)}\right\|^{2}+h \gamma\left\|\widetilde{W}^{(n)}\right\|^{2}\right)
\end{aligned}
$$

which gives (60). The proof is completed.
In the following, we assume that the method (4a), (4b), and $(4 \mathrm{c})$ has generalized stage order $P$; that is, there exists a function $H^{h}(t)$ such that (46) holds. For any $n>0$, we define $\widehat{Y}^{(n)}$ and $\hat{y}^{(n+1)}$ by

$$
\widehat{Y}^{(n)} h C_{11} F\left(t_{n}, \widehat{Y}^{(n)}, Y\left(t_{n}-\tau\right), \widehat{\tilde{Y}}^{(n)}\right)+C_{12} H^{h}\left(t_{n}\right),
$$

$$
\widehat{y}^{(n+1)} h C_{21} F\left(t_{n}, \widehat{Y}^{(n)}, Y\left(t_{n}-\tau\right), \hat{\tilde{Y}}^{(n)}\right)+C_{22} H^{h}\left(t_{n}\right),
$$

where

$$
\widehat{\widetilde{Y}}^{(n)}
$$

$$
\begin{aligned}
= & \left(f\left(t_{n}+c_{1} h-\tau, \bar{Y}_{1}^{(n)}, \bar{Y}_{1}^{(n+1)}, H_{1}^{h}\left(t_{n}+c_{1} h-\tau\right)\right),\right. \\
& f\left(t_{n}+c_{2} h-\tau, \bar{Y}_{2}^{(n)}, \bar{Y}_{2}^{(n+1)}, H_{1}^{h}\left(t_{n}+c_{2} h-\tau\right)\right), \ldots, \\
& f\left(t_{n}+c_{s} h-\tau, \bar{Y}_{s}^{(n)}, \bar{Y}_{s}^{(n+1)}, H_{1}^{h}\left(t_{n}+c_{s} h-\tau\right)\right), \\
& f\left(t_{n}+c_{1} h-h-\tau, \bar{Y}_{1}^{(n-1)}, \bar{Y}_{1}^{(n)},\right. \\
& \left.H_{1}^{h}\left(t_{n}+c_{1} h-h-\tau\right)\right), \ldots, \\
& \left.f\left(t_{n}+c_{s} h-h-\tau, \bar{Y}_{s}^{(n-1)}, \bar{Y}_{s}^{(n)}, H_{1}^{h}\left(t_{n}+c_{s} h-h-\tau\right)\right)\right) .
\end{aligned}
$$

Theorem 16. Suppose that the method (4a), (4b), and (4c) is diagonally stable and its generalized stage order is $P$. Then there exist constants $p_{5}$ and $h_{2}$ such that

$$
\begin{aligned}
& \sum_{k=1}^{n}\left\|\bar{Y}^{(k)}-Y\left(t_{k}-\tau\right)\right\|^{2} \\
& \leq p_{4}\left(\sum_{k=1}^{n}\left\|\zeta^{(k)}-H^{h}\left(t_{k}\right)\right\|^{2}+n h^{(p+1)}+n h^{(v+\mu+1)}\right. \\
&\left.\quad+\sum_{k=-m+1}^{0}\left\|Y^{(k)}-Y\left(t_{k}\right)\right\|^{2}\right), \quad h \in\left(0, h_{2}\right] .
\end{aligned}
$$

Proof. It follows from (6) that

$$
\begin{aligned}
& \left\|\bar{Y}_{j}^{(k)}-y\left(t_{k}+c_{j} h-\tau\right)\right\| \\
& \leq\left\|\sum_{i=-\mu}^{v} \widetilde{L}_{i}(\delta)\left(Y_{j}^{(k-m+i)}-y\left(t_{k-m+i}+c_{j} h\right)\right)\right\| \\
& \quad+\left\|\sum_{i=-\mu}^{v} \widetilde{L}_{i}(\delta) y\left(t_{k-m+i}+c_{j} h\right)-y\left(t_{k-m}+c_{j} h+\delta h\right)\right\| .
\end{aligned}
$$


From the remainder estimation of Lagrange interpolation formula, we have

$$
\begin{aligned}
& \left\|\sum_{i=-\mu}^{v} \widetilde{L}_{i}(\delta) y\left(t_{k-m+i}+c_{j} h\right)-y\left(t_{k-m}+c_{j} h+\delta h\right)\right\| \\
& \quad \leq M_{\mu+v+1} h^{\mu+v+1} .
\end{aligned}
$$

Using Cauchy inequality, we further obtain

$$
\begin{gathered}
\left\|\bar{Y}^{(k)}-Y\left(t_{k}-\tau\right)\right\|^{2} \\
\leq 2 s(\mu+v+2)\left(\widetilde{L}_{0}^{2} \sum_{i=-\mu}^{v}\left\|Y^{(k-m+i)}-Y\left(t_{k-m+i}\right)\right\|^{2}\right. \\
\left.+M_{\mu+v+1} h^{(v+\mu+1)}\right),
\end{gathered}
$$

where $\widetilde{L}_{0}^{2}=\max _{-\mu \leq i \leq v} \operatorname{Sup}_{x \in[0,1]}\left|\widetilde{L}_{i}(x)\right|$. Hence, there exists a constant $d_{1}$ such that

$$
\begin{aligned}
& \sum_{k=1}^{n}\left\|\bar{Y}^{(k)}-Y\left(t_{k}-\tau\right)\right\|^{2} \\
& \quad \leq d_{1}\left(\sum_{k=-\mu-m+1}^{n}\left\|Y^{(k)}-Y\left(t_{k}\right)\right\|^{2}+n h^{2(\mu+v+1)}\right) .
\end{aligned}
$$

On the other hand, a combination of (41a) and (47a) leads to

$$
\begin{aligned}
& Y^{(k)}-Y\left(t_{k}\right) \\
& =h C_{11}\left(F\left(t_{k}, Y^{(k)}, \bar{Y}^{(k)}, \tilde{Y}^{(k)}\right)\right. \\
& \left.\quad-F\left(t_{k}, Y\left(t_{k}\right), Y\left(t_{k}-\tau\right), \tilde{Y}\left(t_{k}-\tau\right)\right)\right) \\
& +C_{12}\left(y^{(k-1)}-H^{h}\left(t_{k}-h\right)\right)-\Delta^{h}\left(t_{k}\right) .
\end{aligned}
$$

It follows from Theorem 14 that

$$
\begin{aligned}
&\left\|Y^{(k)}-Y\left(t_{k}\right)\right\| \\
&=p_{2}\left\{\left\|C_{12}\left(y^{(k-1)}-H^{h}\left(t_{k}-h\right)-\Delta^{h}\left(t_{k}\right)\right)\right\|\right. \\
&\left.+h\left\|\bar{Y}^{(k)}-Y\left(t_{k}-\tau\right)\right\|+h\left\|\widetilde{Y}^{(k)}-\tilde{Y}\left(t_{k}-\tau\right)\right\|\right\},
\end{aligned}
$$

which on substitution into (72) gives

$$
\begin{aligned}
& \sum_{k=1}^{n} \| \bar{Y}^{(k)}- Y\left(t_{k}-\tau\right) \|^{2} \\
& \leq d_{1}\left(p_{1}^{2} h^{2} \sum_{k=1}^{n}\left\|\bar{Y}^{(k)}-Y\left(t_{k}-\tau\right)\right\|^{2}\right. \\
&+p_{1}^{2} h^{2} \sum_{k=1}^{n}\left\|\widetilde{Y}^{(k)}-\tilde{Y}\left(t_{k}-\tau\right)\right\|^{2} \\
&+p_{1}^{2}\left\|C_{12}\right\|^{2} \sum_{k=1}^{n}\left\|y^{(k-1)}-H^{h}\left(t_{k-1}\right)\right\|^{2} \\
&+p_{1}^{2}\left\|C_{12}\right\|^{2}\left\|\Delta^{h}\left(t_{k}\right)\right\|^{2} \\
&\left.+n h^{2(\mu+v+1)}+\sum_{k=-\mu-m+1}^{0}\left\|Y^{(k)}-Y\left(t_{k}\right)\right\|^{2}\right) .
\end{aligned}
$$

Therefore, there exist $p_{4}$ and $h_{2}$ such that (68) holds. The proof of Theorem 16 is completed.

Theorem 17. Suppose that method (4a), (4b), and (4c) is algebraically stable and diagonally stable and its generalized stage order is $p$. Then the method with interpolation procedure (6) is D-convergent of order at least $\min \{p, \mu+v+1\}$.

Proof. In view of (41a), (41b), (66a), and (66b), it follows from Theorem 15 that

$$
\begin{aligned}
& \left\|y^{(n)}-\widehat{y}^{(n)}\right\|_{G}^{2} \\
& \leq p_{3} h\left(\left\|Y^{(n)}-\widehat{Y}^{(n)}\right\|^{2}+\left\|\bar{Y}^{(n)}-Y\left(t_{n}-\tau\right)\right\|^{2}\right. \\
& \left.\quad+\left\|\tilde{Y}^{(n)}-\tilde{Y}\left(t_{n}-\tau\right)\right\|^{2}\right)+\left\|y^{(n-1)}-H^{h}\left(t_{n-1}\right)\right\|_{G}^{2}
\end{aligned}
$$

Using Theorem 14, we have

$$
\begin{aligned}
&\left\|Y^{(n)}-\widehat{Y}^{(n)}\right\| \\
& \leq p_{1}\left\{\left\|C_{12}\right\|\left\|y^{(n-1)}-H^{h}\left(t_{n-1}\right)\right\|\right. \\
&\left.+h\left\|\bar{Y}^{(n)}-Y\left(t_{n}-\tau\right)\right\|+h\left\|\tilde{Y}^{(n)}-\tilde{Y}\left(t_{n}-\tau\right)\right\|\right\}, \\
& h \in\left(0, h_{1}\right],
\end{aligned}
$$

which on substitution into (76) gives

$$
\begin{aligned}
& \left\|y^{(n)}-\widehat{y}^{(n)}\right\|_{G}^{2} \\
& \leq p_{3} h\left(1+2 p_{1}^{2} h^{2}\right)\left(1+2 s r^{2} q^{2}\right) \\
& \quad \times\left\|\bar{Y}^{(n)}-Y\left(t_{n}-\tau\right)\right\|^{2}+\left(1+\frac{2 h p_{3} p_{1}^{2}\left\|C_{12}\right\|^{2}}{\lambda_{2}}\right) \\
& \quad \times\left\|y^{(n-1)}-H^{h}\left(t_{n-1}\right)\right\|_{G}^{2}, \quad h \in\left(0, h_{1}\right],
\end{aligned}
$$


where $\lambda_{2}$ is the minimum characteristic value of $G$. On the other hand,

$$
\begin{aligned}
\left\|y^{(n)}-H^{h}\left(t_{n}\right)\right\|_{G}^{2} \leq & \left\|y^{(n)}-\widehat{y}^{(n)}\right\|_{G}^{2}+\left\|\widehat{y}^{(n)}-H^{h}\left(t_{n}\right)\right\|_{G}^{2} \\
& +2\left\|y^{(n)}-\widehat{y}^{(n)}\right\|_{G}\left\|\hat{y}^{(n)}-H^{h}\left(t_{n}\right)\right\|_{G} \\
\leq & (1+h)\left\|y^{(n)}-\widehat{y}^{(n)}\right\|_{G}^{2} \\
& +\left(1+\frac{1}{h}\right)\left\|\hat{y}^{(n)}-H^{h}\left(t_{n}\right)\right\|_{G}^{2} .
\end{aligned}
$$

In view of (47a), (47b), (48a), (66a), and (66b), the application of Theorem 14 leads to

$$
\left\|\hat{y}^{(n)}-H^{h}\left(t_{n}\right)\right\| \leq p_{2}\left(\left\|\Delta^{h}\left(t_{n}\right)\right\|+\left\|\delta^{h}\left(t_{n}\right)\right\|\right), \quad h \in\left(0, \bar{h}_{0}\right],
$$

which gives

$$
\begin{aligned}
& \left\|\hat{y}^{(n)}-H^{h}\left(t_{n}\right)\right\|_{G}^{2} \\
& \quad \leq \lambda_{1} p_{1}^{2}\left(\left\|\Delta^{h}\left(t_{n}\right)\right\|+\left\|\delta^{h}\left(t_{n}\right)\right\|\right)^{2}, \quad h \in\left(0, \bar{h}_{0}\right],
\end{aligned}
$$

where $\lambda_{1}$ denotes the maximum eigenvalue of the matrix $G$. A combination of (46) and (76)-(81) leads to

$$
\begin{aligned}
& \left\|y^{(n)}-H^{h}\left(t_{n}\right)\right\|_{G}^{2} \\
& \quad \leq\left(1+h \gamma_{1}\right)\left\|y^{(n-1)}-H^{h}\left(t_{n-1}\right)\right\|_{G}^{2} \\
& \quad+\gamma_{2} h\left\|\bar{Y}^{(n)}-Y\left(t_{n}-\tau\right)\right\|^{2}+\gamma_{3} h^{2 p+1}, \quad h \in\left(0, h_{3}\right],
\end{aligned}
$$

where

$$
\begin{gathered}
h_{3}=\min \left\{\bar{h}_{0}, h_{1}\right\} \leq 1, \\
\gamma_{1}=1+\frac{4 p_{3} p_{1}^{2}\left\|C_{12}\right\|^{2}}{\lambda_{2}}, \\
\gamma_{2}=2 p_{3}\left(1+2 p_{1}^{2}\right)\left(1+2 s r^{2} q^{2}\right), \\
\gamma_{3}=8 \lambda_{1} p_{0}^{2} p_{2}^{2} .
\end{gathered}
$$

Therefore,

$$
\begin{aligned}
& \left\|y^{(n)}-H^{h}\left(t_{n}\right)\right\|_{G}^{2} \\
& \leq\left\|y^{(0)}-H^{h}\left(t_{0}\right)\right\|_{G}^{2} \\
& \quad+h \sum_{i=1}^{n}\left(\gamma_{1}\left\|y^{(i-1)}-H^{h}\left(t_{i-1}\right)\right\|_{G}^{2}+\gamma_{2}\left\|\bar{Y}^{(i)}-Y\left(t_{i}-\tau\right)\right\|^{2}\right) \\
& \quad+\gamma_{3} t_{n} h^{2 p}, \quad h \in\left(0, h_{3}\right] .
\end{aligned}
$$

Considering Theorem 16, we further obtain

$$
\begin{aligned}
& \left\|y^{(n)}-H^{h}\left(t_{n}\right)\right\|_{G}^{2} \\
& \leq\left\|y^{(0)}-H^{h}\left(t_{0}\right)\right\|_{G}^{2}+h\left(\gamma_{1}+\gamma_{2} p_{4}\right) \\
& \quad \times \sum_{i=0}^{n-1}\left\|y^{(i)}-H^{h}\left(t_{i}\right)\right\|_{G}^{2}+\gamma_{3} t_{n} h^{2 p} \\
& +\gamma_{2} p_{4} t_{n}\left(h^{2(p+1)}+h^{2(\mu+v+1)}\right)+p_{4} \gamma_{2} h \\
& \quad \times \sum_{k=-\mu-m+1}^{0}\left\|Y^{(k)}-Y\left(t_{k}\right)\right\|^{2}, \quad h \in\left(0, h_{0}\right],
\end{aligned}
$$

where $h_{0}=\min \left\{h_{2}, h_{3}\right\} \leq 1$. Using discrete Bellman inequality, we have

$$
\begin{aligned}
\left\|y^{(n)}-H^{h}\left(t_{n}\right)\right\|_{G}^{2} \leq & \left(\left\|y^{(0)}-H^{h}\left(t_{0}\right)\right\|_{G}^{2}+p_{4} \gamma_{2} h\right. \\
& \times \sum_{k=-\mu-m+1}^{0}\left\|Y^{(k)}-Y\left(t_{k}\right)\right\|^{2} \\
& \left.+\left(\gamma_{3}+\gamma_{2} p_{4}\right) t_{n} h^{2 p}+\gamma_{2} p_{4} t_{n} h^{2(\mu+v+1)}\right) \\
& \times \exp \left(\left(\gamma_{1}+\gamma_{2} p_{4}\right) t_{n}\right), \quad h \in\left(0, h_{0}\right] .
\end{aligned}
$$

Considering $\left\|H(t)-H^{h}\left(t_{n}\right)\right\| \leq p_{0} h^{p}$, we obtain

$$
\begin{aligned}
\left\|y^{(n)}-H\left(t_{n}\right)\right\| & \leq\left\|y^{(n)}-H^{h}\left(t_{n}\right)\right\|+\left\|H^{h}\left(t_{n}\right)-H\left(t_{n}\right)\right\| \\
& \leq\left\|y^{(n)}-H^{h}\left(t_{n}\right)\right\|+p_{0} h^{p} .
\end{aligned}
$$

Considering (87) and (88), we can easily conclude that method (4a), (4b), and (4c) with interpolation procedure (6) is $D$-convergent of order at least $\min \{p, \mu+v+1\}$. The proof is completed.

\section{Numerical Experiments}

Consider the following nonlinear neutral delay differential equations:

$$
\begin{aligned}
& y^{\prime}(t) \\
& =-y(t)+0.9 \cos \left(y(t-1)+y^{\prime}(t-1)\right) \\
& +\cos (t) e^{-t}-0.9 \cos \left(\cos (t-\tau) e^{-(t-\tau)}\right), \quad 10 \geq t \geq 0 \\
& y(t)=e^{-t} \sin (t), \quad-1 \leq t \leq 0,
\end{aligned}
$$




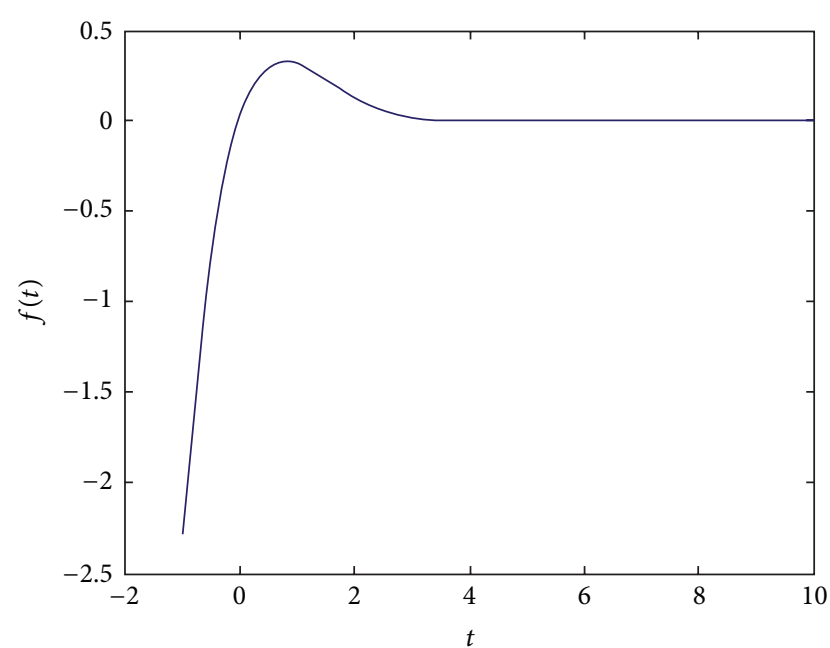

FIgURE 1: The numerical solution of (92) for (89) with $h=0.1$. and its perturbed problem

$$
\begin{aligned}
& z^{\prime}(t) \\
& =-z(t)+0.9 \cos \left(z(t-1)+z^{\prime}(t-1)\right)+\cos (t) e^{-t} \\
& -0.9 \cos \left(\cos (t-\tau) e^{-(t-\tau)}\right), \quad 10 \geq t \geq 0, \\
& z(t)=e^{-t} \sin (t)+0.2, \quad-1 \leq t \leq 0 .
\end{aligned}
$$

We can calculate $\alpha=-1, \beta=0.9, \gamma=0.9$, and $q=0$. Equation (89) has a unique true solution:

$$
y(t)=e^{-t} \sin (t), \quad t \geq-1
$$
system (89).

\begin{tabular}{lcccc}
\hline$m$ & 5 & 10 & 20 & 40 \\
Convergence order & 4.0012 & 3.9880 & 3.9864 & 3.9860 \\
\hline
\end{tabular}

Apply the two-step Runge-Kutta method induced by the GL method in [12]

\begin{tabular}{c|c|c|c}
$a_{11}$ & $a_{12}$ & $a_{21}$ & $a_{22}$ \\
\hline 0.47790690818421 & 0.87165188291653 & -0.08663699023763 & 0.50361252124048 \\
\hline$b_{1}$ & $b_{2}$ & $\tilde{b}_{1}$ & $\tilde{b}_{2}$ \\
\hline 0.95532987568936 & 0.79063681672548 & $2 \sqrt{15}-7$ & $8-2 \sqrt{15}$ \\
\hline \multirow{2}{*}{$c_{1}$} & $c_{2}$ & \\
\cline { 2 - 4 } & 1.59379439197950 & 0.44316674917114 & \\
\cline { 2 - 4 } & $\theta$ & $\theta$
\end{tabular}

to (89) and (90), $\tilde{Y}_{j}^{(n)}$ is computed by the Lagrange interpolation procedure with $n=5$ (see Figures 1 and 2 and Table 1).

It is obvious that the corresponding method for NDDEs is stable and convergent, and the convergence order is $\min \{4,5\}$.

\section{Conclusions}

In this paper we gave the stability and convergence results of two-step Runge-Kutta methods with linear interpolation procedure for solving nonlinear NDDEs (1). First, we gave the definitions of $(k, l)$-algebraically stable, algebraically stable and diagonally stable. Then we proved that if a TSRK method is $(k, l)$-algebraically stable, $k<1$ and $d_{j}>0$ $j=1,2, \ldots, s$, then the method with linear interpolation procedure is weak $\operatorname{GAR}(l)$-stable. We also proved that if a TSRK is algebraically stable and diagonally stable and its generalized stage order is $p$, then the method with interpolation procedure is $D$-convergent of order at least $\min \{p, \mu+v+1\}$.

We believe that the results presented in this paper can be extended to other general NDDEs. However, it is difficult to extend these results to more general neutral functional differential equations. Results extending the results presented in this paper to more general neutral functional differential equations and other delay differential equations such as delay integral differential equations will be discussed elsewhere. 


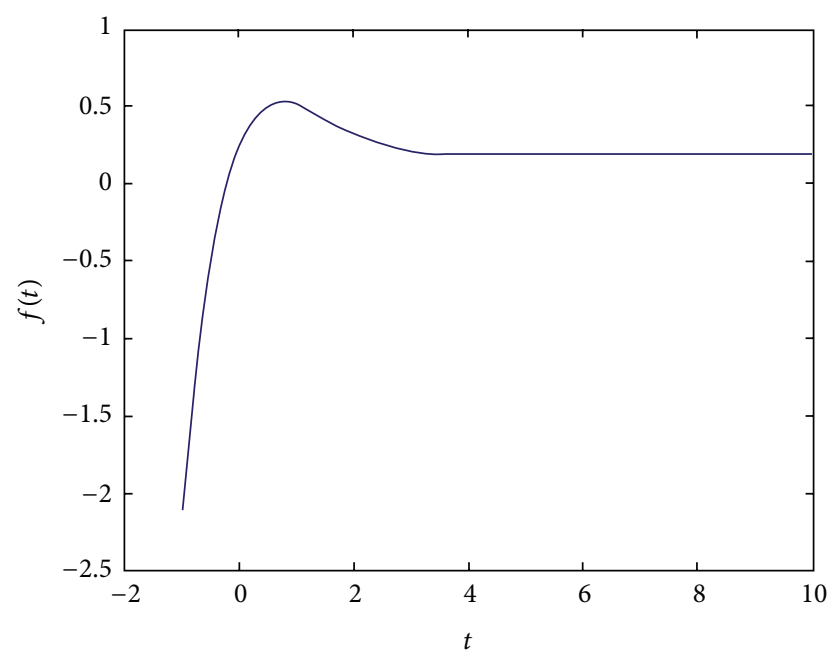

FIGURE 2: The numerical solution of (92) for (90) with $h=0.1$.

\section{Acknowledgments}

This work was supported by the Education Foundation of Heilongjiang Province of China (12523039), the Natural Science Foundation of Heilongjiang Province of China (QC2011C020), and the Doctor Foundation of Heilongjiang Institute of Technology (2012BJ27).

\section{References}

[1] T. E. Wheldon, J. Kirk, and H. M. Finlay, "Cyclical granulopoiesis in chronic granulocytic leukemia: a simulation study," Blood, vol. 43, no. 3, pp. 379-387, 1974.

[2] A. Ruehli, U. Miekkala, A. Bellen, and H. Heeb, "Stable time domain solutions for EMC problems using PEEC circuit models," in Proceedings of the IEEE International Symposium on Electromagnetic Compatibility, pp. 371-376, August 1994.

[3] R. K. Brayton, "Small signal stability criterion for networks containing lossless transmission lines," IBM Journal of Research and Development, vol. 12, pp. 431-440, 1968.

[4] N. Guglielmi, "Inexact Newton methods for the steady state analysis of nonlinear circuits," Mathematical Models and Methods in Applied Sciences, vol. 6, no. 1, pp. 43-57, 1996.

[5] Y. Kuang, Delay Differential Equations with Applications in Population Dynamics, vol. 191, Academic Press, Boston, Mass, USA, 1993.

[6] A. Bellen, N. Guglielmi, and M. Zennaro, "On the contractivity and asymptotic stability of systems of delay differential equations of neutral type," BIT Numerical Mathematics, vol. 39, no. 1, pp. 1-24, 1999.

[7] L. P. Sun, "Stability analysis for linear delay differential equations and numerical examples," Applied Mathematics B, vol. 18, no. 4, pp. 390-402, 2003.

[8] J. X. Kuang, J. X. Xiang, and H. J. Tian, “The asymptotic stability of one-parameter methods for neutral differential equations," BIT, vol. 34, no. 3, pp. 400-408, 1994.

[9] L. Qiu, B. Yang, and J. Kuang, "The NGP-stability of RungeKutta methods for systems of neutral delay differential equations," Numerische Mathematik, vol. 81, no. 3, pp. 451-459, 1999.
[10] H. J. Tian, J. X. Kuang, and L. Qiu, “The stability of linear multistep methods for linear systems of neutral differential equations," Journal of Computational Mathematics, vol. 19, no. 2, pp. 125-130, 2001.

[11] C. Zhang and S. Zhou, "The asymptotic stability of theoretical and numerical solutions for systems of neutral multidelaydifferential equations," Science in China, Series A, vol. 41, no. 11, pp. 504-515, 1998.

[12] A. Bellen and M. Zennaro, Numerical Methods for Delay Differential Equations, Oxford University Press, Oxford, UK, 2003.

[13] L. Torelli, "Stability of numerical methods for delay differential equations," Journal of Computational and Applied Mathematics, vol. 25, no. 1, pp. 15-26, 1989.

[14] L. Torelli, "A sufficient condition for GPN-stability for delay differential equations," Numerische Mathematik, vol. 59, no. 1, pp. 311-320, 1991.

[15] A. Bellen, "Contractivity of continuous Runge-Kutta methods for delay differential equations," Applied Numerical Mathematics, vol. 24, no. 2-3, pp. 219-232, 1997.

[16] A. Bellen and M. Zennaro, "Strong contractivity properties of numerical methods for ordinary and delay differential equations," Applied Numerical Mathematics, vol. 9, no. 3-5, pp. 321346, 1992.

[17] M. Zennaro, "Contractivity of Runge-Kutta methods with respect to forcing terms," Applied Numerical Mathematics, vol. 11, no. 4, pp. 321-345, 1993.

[18] T. Koto, "The stability of natural Runge-Kutta methods for nonlinear delay differential equations," Japan Journal of Industrial and Applied Mathematics, vol. 14, no. 1, pp. 111-123, 1997.

[19] A. Bellen, N. Guglielmi, and M. Zennaro, "Numerical stability of nonlinear delay differential equations of neutral type," Journal of Computational and Applied Mathematics, vol. 125, no. 1-2, pp. 251-263, 2000.

[20] Z. Jackiewicz, "One-step methods of any order for neutral functional differential equations," SIAM Journal on Numerical Analysis, vol. 21, no. 3, pp. 486-511, 1984.

[21] Z. Jackiewicz, "Quasilinear multistep methods and variable step predictor-corrector methods for neutral functional differential equations," SIAM Journal on Numerical Analysis, vol. 23, pp. 423-452, 1986.

[22] Z. Jackiewicz, M. Kwapisz, and E. Lo, "Waveform relaxation methods for functional-differential systems of neutral type," Journal of Mathematical Analysis and Applications, vol. 207, no. 1, pp. 255-285, 1997.

[23] R. Yang, H. Gao, and P. Shi, "Novel robust stability criteria for stochastic Hopfield neural networks with time delays," IEEE Transactions on Systems, Man, and Cybernetics, Part B, vol. 39, pp. 467-474, 2009.

[24] R. Yang, Z. Zhang, and P. Shi, "Exponential stability on stochastic neural networks with discrete interval and distributed delays," IEEE Transactions on Neural Networks, vol. 21, no. 1, pp. 169-175, 2010.

[25] L. P. Liu, "Robust stability for neutral time-varying delay systems with non-linear perturbations," International Journal of Innovative Computing, Information and Control, vol. 17, no. 10, pp. 5749-5760, 2011.

[26] A. Tanikawa, "On stability of the values of random zerosum games," International Journal of Innovative Computing, Information and Control, vol. 7, no. 1, pp. 133-140, 2011. 
[27] M. Basin and D. Calderon-Alyarez, "Delay-dependent stability for vector nonlinear stochastic systems with multiple delays," International Journal of Innovative Computing, Information and Control, vol. 7, no. 4, pp. 1565-1576, 2011.

[28] H. J. Stetter, "Numerische Losung von Differential gleichungen mit nacheilendem Argument," ZAMM Journal of Applied Mathematics and Mechanics, vol. 45, pp. 79-80, 1965.

[29] H. J. Oberle and H. J. Pesch, "Numerical treatment of delay differential equations by Hermite Interpolation," Numerische Mathematik, vol. 37, no. 2, pp. 235-255, 1981.

[30] M. Zennaro, "Natural continuous extensions of Runge-Kutta methods," Mathematics of Computation, vol. 46, no. 173, pp.119133, 1986.

[31] C. M. Huang, "Algebraic stability of hybrid methods," Mathematica Numerica Sinica, vol. 17, no. 3, pp. 67-74, 1995.

[32] S. F. Li, Theory of Computational Methods For Stiff Differential Equations, Hunan Science and Technology Publisher, Changsha, China, 1997. 


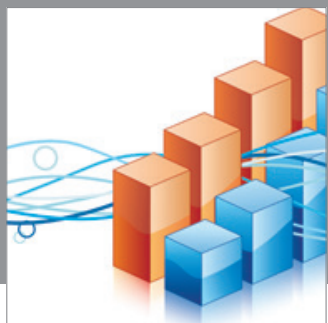

Advances in

Operations Research

mansans

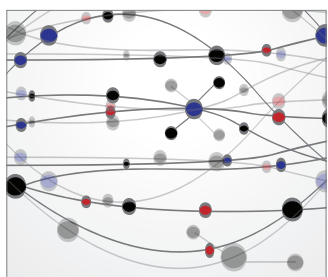

The Scientific World Journal
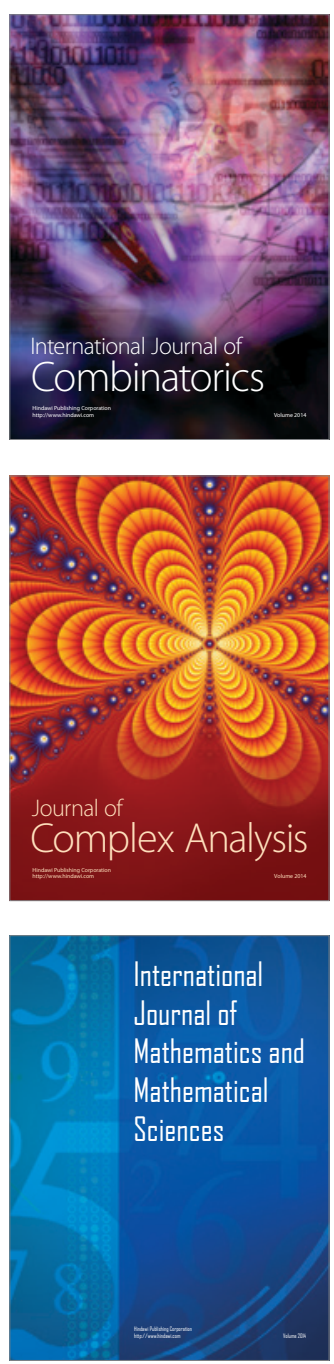
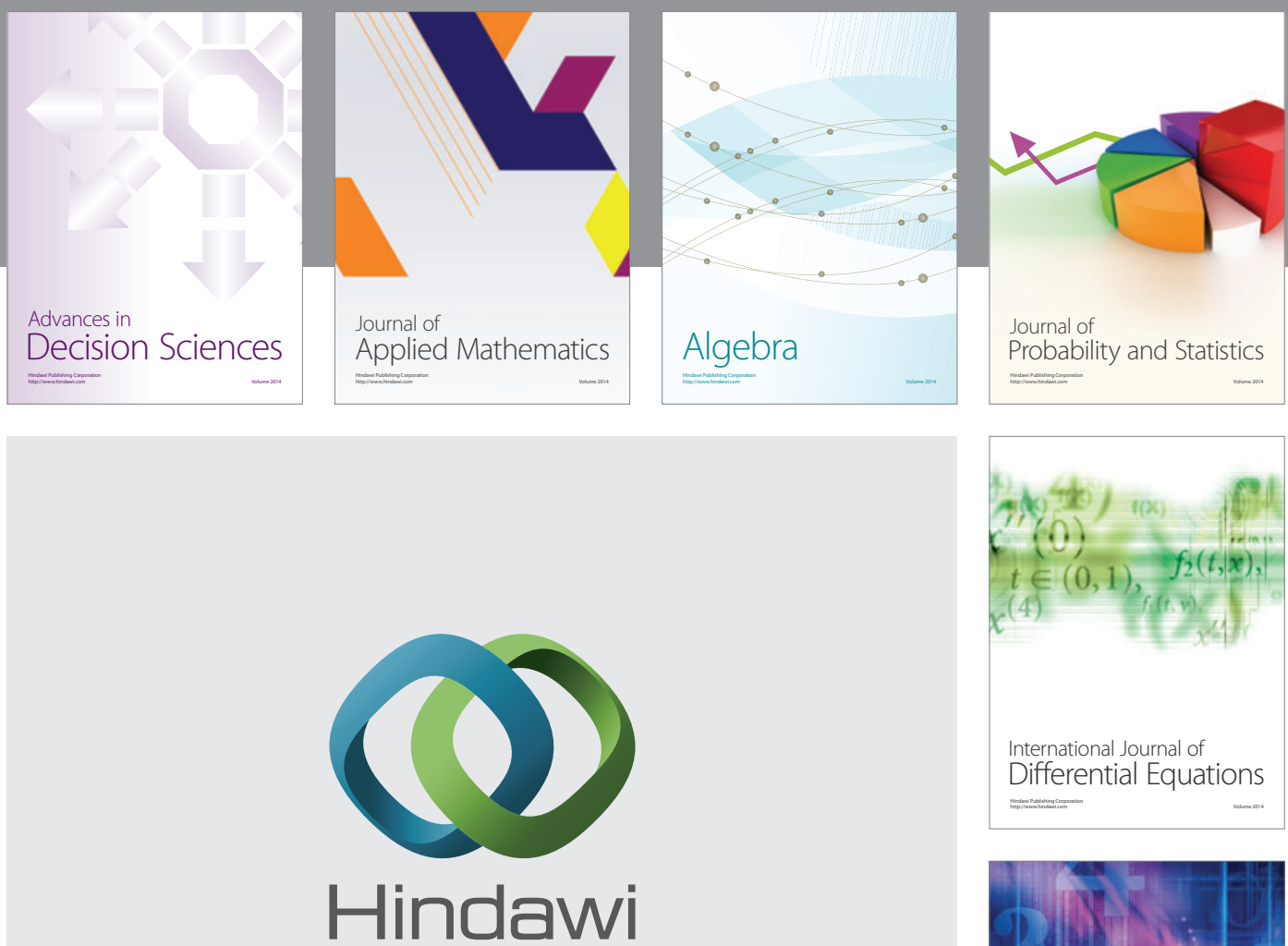

Submit your manuscripts at http://www.hindawi.com
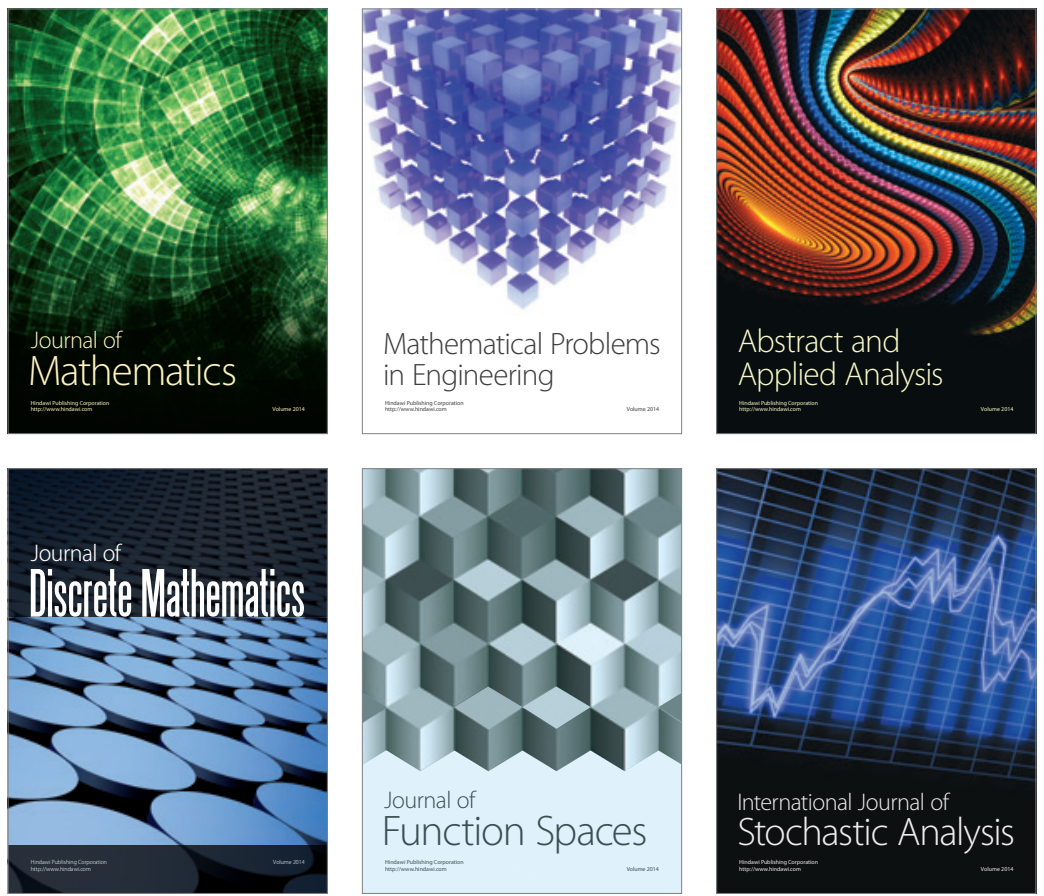

Journal of

Function Spaces

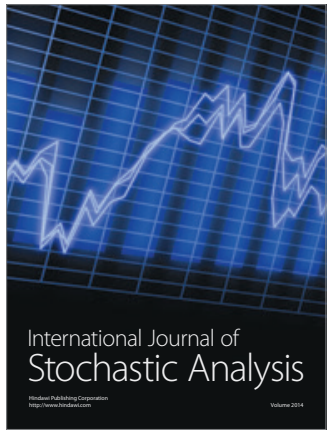

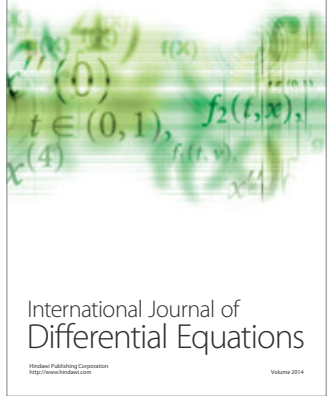
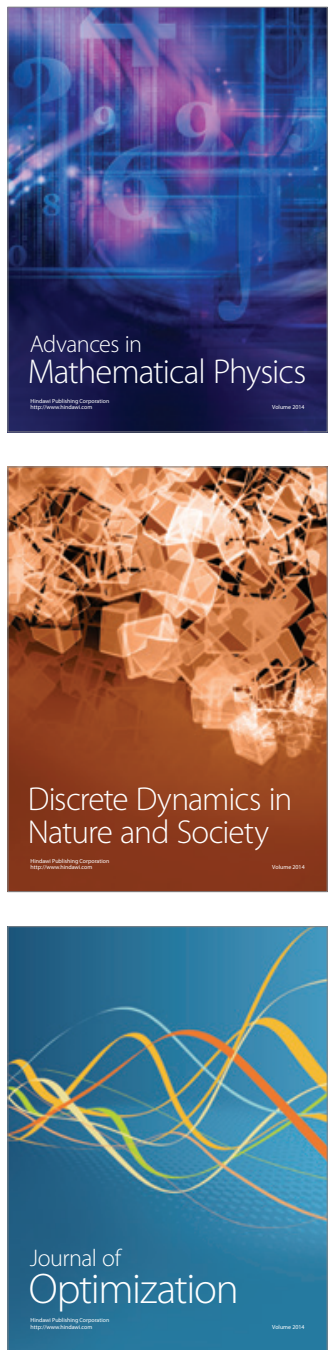